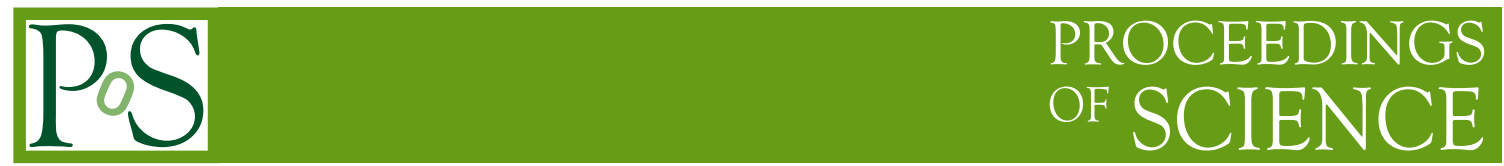

\title{
Measurements of $\bar{t} \bar{t}+X$ in ATLAS and CMS
}

\section{Francisco Yumiceva ${ }^{a, *}$}

a Florida Institute of Technology,

150 West University Blvd, Melbourne, FL 32950, USA

E-mail: fyumiceva@fit.edu

We summarize the recent results from the ATLAS and CMS collaborations on the measurements of the cross sections of $\overline{\mathrm{t}}+\mathrm{W}, \mathrm{t} \overline{\mathrm{t}}+\mathrm{Z}, \mathrm{t} \overline{\mathrm{t}}+\gamma$, and $\overline{\mathrm{t}} \mathrm{t} \overline{\mathrm{b}}$ processes using data collected in proton-proton collisions at the LHC at $\sqrt{s}=8$ and $13 \mathrm{TeV}$.

The Eighth Annual Conference on Large Hadron Collider Physics-LHCP2020

25-30 May, 2020

online

${ }^{*}$ Speaker 


\section{1. $t \bar{t}+W$ Production}

The current published results on the measurement of the $\bar{t}+\mathrm{W}$ cross section use a data sample with a total luminosity of $36 \mathrm{fb}^{-1}$ collected by each experiment. The analyses apply a single lepton triggers and separate the data and simulated samples into several signal regions depending on the lepton electric charge and flavor, number of jets and $b$ tagged jets, see Table 1 . In the ATLAS analysis [1], the analysis applies a MVA to distinguish the prompt lepton from the nonprompt $\ell$ originating from hadron decays, $\gamma$ conversions or misidentified jets. A second MVA is used to suppress the misidentified electron charge in the SS channel. Control regions (CR) are constructed to estimate the following largest backgrounds. The charge-flip CR has the charge requirement removed. The fake leptons CR uses the matrix method or tight-to-loose likelihood technique to estimate this background contribution. Several orthogonal samples are produced by inverting the lepton charge or flavor, and the number of jets. In the CMS analysis [2], a BDT is constructed using the variables $N_{j}, N_{b}, H_{T}, p_{T}^{m i s s}, p_{T}^{\ell}, M_{T}$, and $\Delta R(\ell, j$. The contribution from fake leptons is estimated using the tight-to-loose method with a corrected lepton $p_{T}$ as a function of lepton $p_{T}$ and $\eta$. The charge misidentification and $\mathrm{WZ}$ diboson contributions are also extracted with data driven methods. Additional backgrounds are taken from the simulation predictions, $t(t) X=t t H, t q Z$, rare=multibosons. The largest systematic uncertainties are from the data-driven background statistics and the modeling of the $\bar{t}+\mathrm{W}$ in the case of the ATLAS analysis while the nonprompt and ttX background estimations and the statistical uncertainty in the nonprompt background method are the largest in the CMS result. ATLAS measures a cross section of $\sigma_{t t W}=0.87 \pm 0.13$ (stat) \pm 0.14 (syst) pb and CMS measures $\sigma_{t t W}=0.77_{-0.11}^{+0.12}$ (stat) ${ }_{-0.12}^{+0.13}$ (syst) pb, and separated by W charge $\sigma_{t t W^{+}}=0.58 \pm 0.09$ (stat) $)_{-0.08}^{+0.09}(\mathrm{syst}) \mathrm{pb}$ and $\sigma_{t t W^{-}}=0.19 \pm 0.07(\mathrm{stat}) \pm$ 0.06(syst) pb

\begin{tabular}{|l|c|c|c|}
\hline \multicolumn{2}{|c|}{ Processes $(\ell=\mathrm{e}, \mu)$} & Channel & Signal Region \\
\hline \multirow{2}{*}{$\mathrm{t}+\mathrm{W}$} & $\mathrm{t} \overline{\mathrm{t}} \rightarrow 1 \ell$ & $\mathrm{SS} 2 \ell$ & $12\left(\right.$ sign, flavor, $\left.N_{b}\right)$ \\
& $\mathrm{t} \overline{\mathrm{t}} \rightarrow 2 \ell$ & $3 \ell$ & $8\left(\right.$ sign, $\left.N_{j}, N_{b}\right)$ \\
\hline
\end{tabular}

Table 1: Number of signal regions per $\mathrm{t} \overline{\mathrm{t}}+\mathrm{W}$ processes and lepton flavor $(\ell)$ as a function of charge sign, flavor, number of jets $N_{j}$, and b tagged jets $N_{b}$.
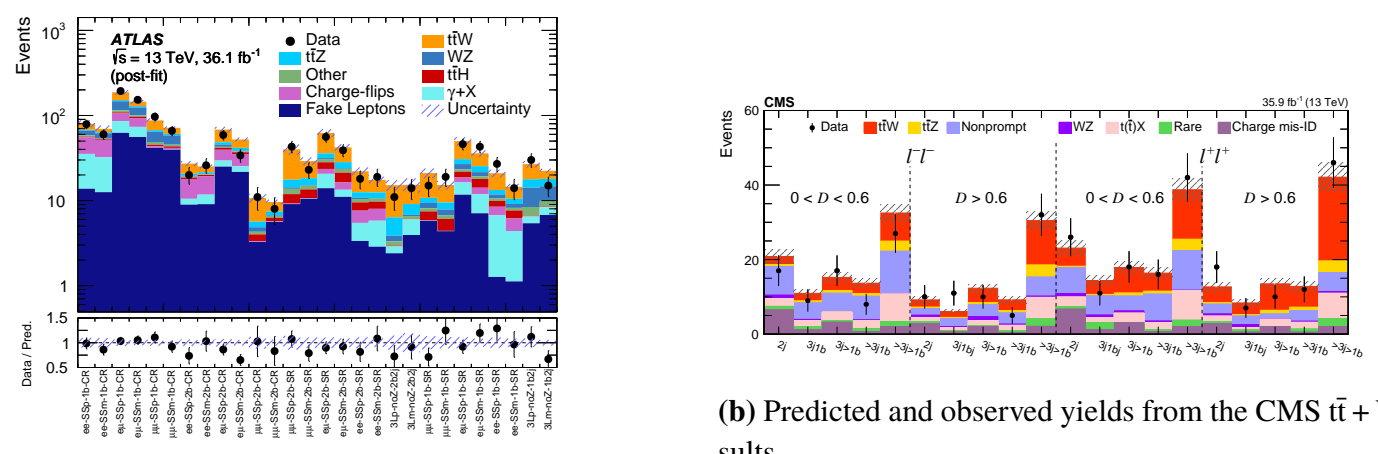

(b) Predicted and observed yields from the CMS $\mathrm{t}+\mathrm{W}$ results.

(a) Event yields from control regions, SS and $3 \ell$ results from the ATLAS collaboration for the $\mathrm{t} \overline{\mathrm{t}}+\mathrm{W}$ analysis. 


\section{2. $t \bar{t}+Z$ Production}

The ATLAS $t \bar{t}+Z$ analysis uses a very similar approach as the $t \bar{t}+W$ analysis using a sample of $36.1 \mathrm{fb}^{-1}$ while CMS has a dedicated analysis with a sample of $77.5 \mathrm{fb}^{-1}$ [3]. The $\overline{\mathrm{t}}+\mathrm{Z}$ decays are classified according to the number of leptons in the final state. Table 2 lists the processes, channels, and signal regions used in the $\bar{t}+\mathrm{Z}$ analyses. The current analyses concentrate on the trilepton and tetralepton final states. Small background contributions from $t(t) X$ where $X=\mathrm{H}, \mathrm{W}$, $\mathrm{WZ}, \mathrm{Zq}, \mathrm{Hq}, \mathrm{HW}, \mathrm{VV}(\mathrm{V}=\mathrm{W}, \mathrm{Z}), \mathrm{t} \overline{\mathrm{t}}$, and rare $=\mathrm{VVV}, \mathrm{X} \gamma$ are taken from simulation. The yield of $\mathrm{WZ}(3 \ell)$ is estimated using a CR with $N_{b}=0$ events. The yield of $\mathrm{ZZ}(4 \ell)$ is estimated using a $C R$ with reconstructed $Z Z$ events. The fake leptons are controlled with a CR that vetoes $Z(3 \ell)$ events. CMS has recently made some improvements to its analysis using more inclusive lepton triggers that combine the single, dilepton, and trilepton triggers. A dedicated MVA is used for the lepton selection which has a $15 \%$ increase in the prompt lepton efficiency and the fake rate is reduced by 2-to- 4 times with respect to the previous CMS selection. ATLAS reports a result of $\sigma_{t t Z}=0.95 \pm 0.08$ (stat) \pm 0.10 (syst) pb

\begin{tabular}{|l|c|c|c|}
\hline \multicolumn{2}{|c|}{ Processes $(\ell=\mathrm{e}, \mu)$} & Channel & Signal Region \\
\hline \multirow{4}{*}{$\mathrm{t}+\mathrm{Z}$} & $\mathrm{t} \overline{\mathrm{t}} \rightarrow 0 \ell$ & OS2 $\ell$ & $4\left(N_{j}, N_{b}\right)$ \\
& $\mathrm{t} \overline{\mathrm{t}} \rightarrow 1 \ell$ & $3 \ell$ & $8\left(\right.$ sign, $\left.N_{j}, N_{b}\right)$ \\
& $\mathrm{t} \overline{\mathrm{t}} \rightarrow 2 \ell$ & $4 \ell$ & 4 (flavor, $\left.N_{j}, N_{b}\right)$ \\
\hline
\end{tabular}

Table 2: Number of signal regions per $\mathrm{t} \overline{\mathrm{t}}+\mathrm{Z}$ processes and lepton flavor $(\ell)$ as a function of charge sign, flavor, number of jets $N_{j}$, and b tagged jets $N_{b}$.
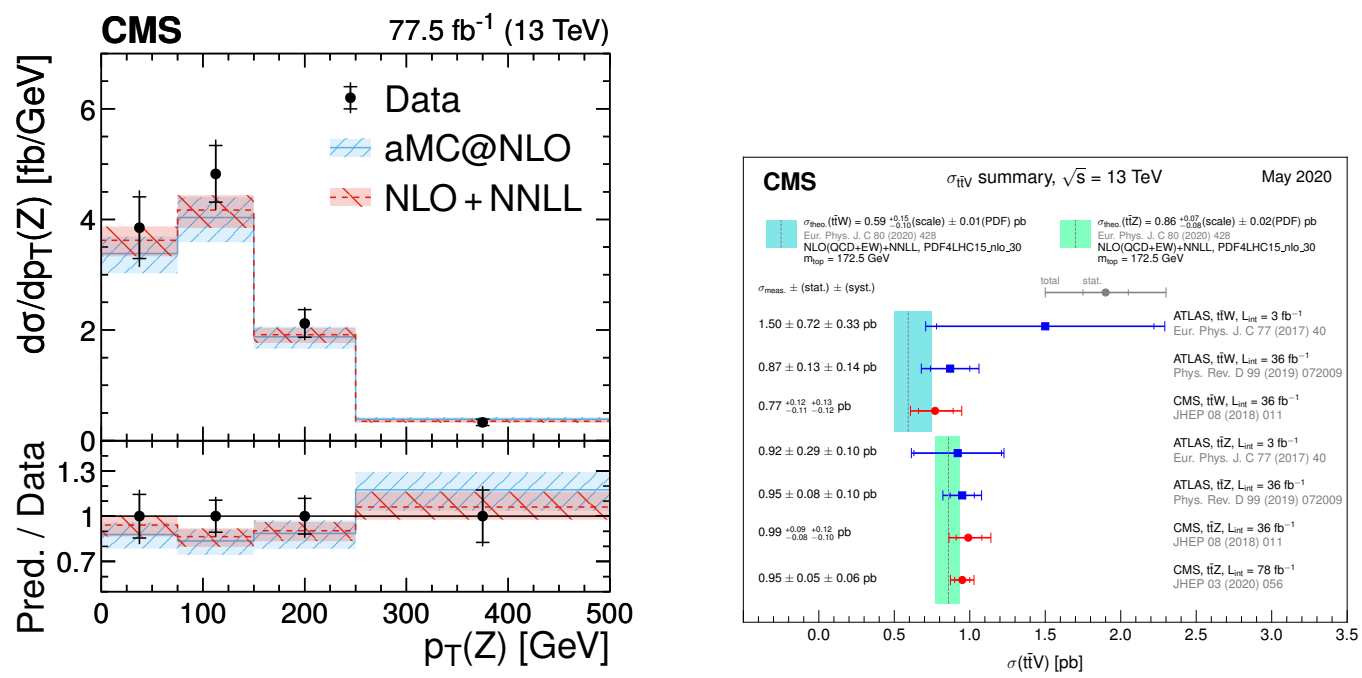

(a) Measured absolute differential $\bar{t}+Z$ production cross (b) Summary of the $\bar{t}+W$ and $\bar{t}+Z$ results from the ATsections in the full phase space as a function of $p_{T}$ of LAS and CMS collaborations compared with the theoretical the $\mathrm{Z}$ boson. The inner (outer) vertical lines indicate the predictions.

statistical (total) uncertainties. The solid histogram shows

the predictions from the MADGRAPH5 AMC@NLO, and

NLO+NNLL accuracy. 


\section{3. $t \bar{t}+\gamma$ Production}

The basis of this analysis is the standard $t \bar{t}$ selection criteria with a procedure to separate the prompt from non-prompt photons. The prompt photon comes directly from the matrix elements. Fake photons or non-prompt photons originate from jet misidentification (a photon from a jet fragmentation or hadron decay) and electron misidentification (an electron missing a track, failed tracking or calorimeter matching, fake photon-conversion vertex, or a photon from a hard electron bremsstrahlung. The cross section is measured in a fiducial phase space defined by a photon with loose kinematic constraints. The largest systematic uncertainties are from the fit procedure to extract the cross section, JES, and MC modeling scales. CMS reports a measurement of $\sigma_{t t \gamma}^{\mathrm{fid}}=127 \pm 27$ fb using $19.7 \mathrm{fb}^{-1}$ at $\sqrt{s}=8 \mathrm{TeV}$ [4], while ATLAS measures $\sigma_{t t \gamma}^{2 \ell}=44.2 \pm 0.9$ (stat) ${ }_{-2.4}^{+2.6}$ (syst) fb using $139 \mathrm{fb}^{-1}$ at $\sqrt{s}=13 \mathrm{TeV}$ using a sample of dilepton e- $\mu$ events $[5,6]$.

\section{4. $\mathrm{t} \overline{\mathrm{t}}+\mathrm{b} \overline{\mathrm{b}}$ Production}

The single lepton analysis uses events with at least 6 jets while the dilepton analysis at least 4 jets. A discriminator is constructed with $b$ tagging jet information. In the case of ATLAS [9], all jets in the event are considered for the training while CMS [8] uses additional jets not associated to the $t \bar{t}$ system. ATLAS single lepton uses a $2 \mathrm{D}$ fit over the $3 \mathrm{rd}$ and 4 th $\mathrm{b}$ tagged jet discriminator and the dilepton uses a 1D fit over the 3rd b tagged jet discriminator. CMS uses a 2D template fit with additional $\mathrm{b}$ tagged jets and extracts the $t \bar{t} j j$ and ratio of $\sigma_{t \bar{t} b b} / \sigma_{t \bar{t} j j}$. The leading systematic uncertainties are from $\mathrm{b}$ tagging, JES, ISR, $t \bar{t} c$ background, and the parton shower. CMS also has a dedicated analysis in the all hadronic channel [7]. It requires at least 8 jets. The QCD is rejected with a MVA built from a quark/gluon likelihood and jet combination matching. A BDT with inputs from a $\chi^{2}$-mass variables and $\mathrm{b}$ tagging discriminators from additional jets. Control regions from the MVA side bands are chosen to control the background. The dominant systematic uncertainties are from $b$ tagging, quark/gluon likelihood, renormalization and factorization scales, and MC statistics. Figures $3 \mathrm{a}$ and $3 \mathrm{~b}$ summarize the results from ATLAS and CMS experiments.

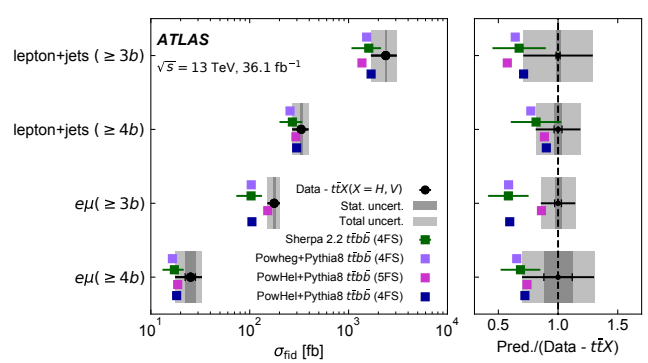

(a) Summary of the t⿱tøb $\bar{b}$ results from ATLAS.

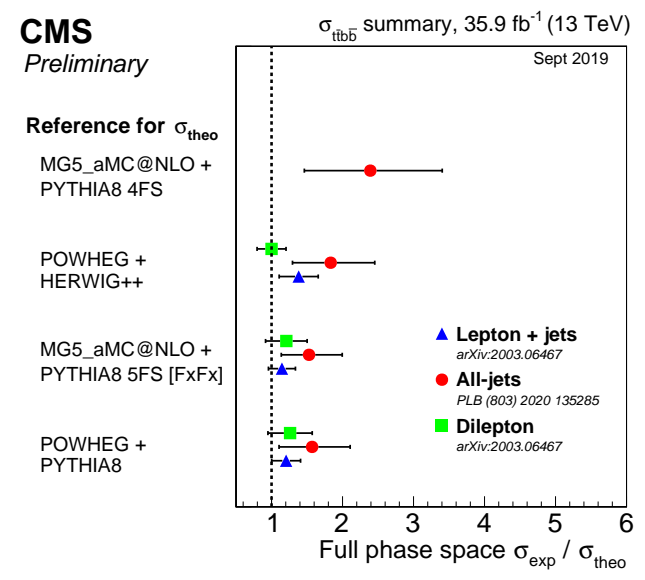

(b) Summary of the CMS measurements of the t⿱t口b $\bar{b}$ processes. 


\section{References}

[1] M. Aaboud et al. [ATLAS], "Measurement of the $t \bar{t} Z$ and $t \bar{t} W$ cross sections in proton-proton collisions at $\sqrt{s}=13 \mathrm{TeV}$ with the ATLAS detector," Phys. Rev. D 99, no.7, 072009 (2019) doi:10.1103/PhysRevD.99.072009 [arXiv:1901.03584 [hep-ex]].

[2] A. M. Sirunyan et al. [CMS], "Measurement of the cross section for top quark pair production in association with a W or Z boson in proton-proton collisions at $\sqrt{s}=13 \mathrm{TeV}$," JHEP $\mathbf{0 8}, 011$ (2018) doi:10.1007/JHEP08(2018)011 [arXiv:1711.02547 [hep-ex]].

[3] A. M. Sirunyan et al. [CMS], "Measurement of top quark pair production in association with a $\mathrm{Z}$ boson in proton-proton collisions at $\sqrt{s}=13 \mathrm{TeV}$," JHEP 03, 056 (2020) doi:10.1007/JHEP03(2020)056 [arXiv:1907.11270 [hep-ex]].

[4] A. M. Sirunyan et al. [CMS], "Measurement of the semileptonic $\overline{\mathrm{t}}+\gamma$ production cross section in pp collisions at $\sqrt{s}=8 \mathrm{TeV}$," JHEP 10, 006 (2017) doi:10.1007/JHEP10(2017)006 [arXiv:1706.08128 [hep-ex]].

[5] G. Aad et al. [ATLAS], "Measurements of inclusive and differential cross-sections of combined $t \bar{t} \gamma$ and $t W \gamma$ production in the $e \mu$ channel at $13 \mathrm{TeV}$ with the ATLAS detector," JHEP 09, 049 (2020) doi:10.1007/JHEP09(2020)049 [arXiv:2007.06946 [hep-ex]].

[6] M. Aaboud et al. [ATLAS], "Measurements of inclusive and differential fiducial cross-sections of $t \bar{t} \gamma$ production in leptonic final states at $\sqrt{s}=13 \mathrm{TeV}$ in ATLAS," Eur. Phys. J. C 79, no.5, 382 (2019) doi:10.1140/epjc/s10052-019-6849-6 [arXiv:1812.01697 [hep-ex]].

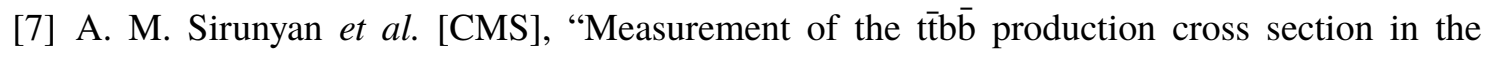
all-jet final state in pp collisions at $\sqrt{s}=13 \mathrm{TeV}$," Phys. Lett. B 803, 135285 (2020) doi:10.1016/j.physletb.2020.135285 [arXiv:1909.05306 [hep-ex]].

[8] A. M. Sirunyan et al. [CMS], "Measurement of the cross section for t⿱t production with additional jets and b jets in pp collisions at $\sqrt{s}=13$ TeV," JHEP 07, 125 (2020) doi:10.1007/JHEP07(2020)125 [arXiv:2003.06467 [hep-ex]].

[9] M. Aaboud et al. [ATLAS], "Measurements of inclusive and differential fiducial crosssections of $t \bar{t}$ production with additional heavy-flavour jets in proton-proton collisions at $\sqrt{s}=13 \mathrm{TeV}$ with the ATLAS detector," JHEP 04, 046 (2019) doi:10.1007/JHEP04(2019)046 [arXiv:1811.12113 [hep-ex]]. 